\title{
Inhabiting the Data Border
}

\author{
ERSELA KRIPA \\ Texas Tech University College of Architecture
}

\section{STEPHEN MUELLER}

Texas Tech University College of Architecture

Just as borders mineralize jurisdictional boundaries, they also create gaps in the availability and interoperability of geospatial data, limiting the ability to forecast crossborder transformations. The geography of borderspace is defined by fragmented, proprietary datasets. Differences in methods, measurements, protocols, and languages leave blindspots for researchers, planners, and designers seeking impacts across a range of fields. Additionally, changing political climates and research agendas affect the availability of comprehensive cross-border environmental data. We present Inhabiting the Data Border, an immersive installation and platform for reflection on working within and across fragmented datascapes, and outline emerging research and initiatives from POST (Project for Operative Spatial Technologies), where we are working to forge mutually-supportive communities and shared environmental, cultural, and geopolitical interests within this context.

\section{BARRIERS TO TRANSNATIONAL GEOSPATIAL INFORMATION PARITY AT THE U.S./MEXICO BORDER}

Open access to robust geospatial information can be a decisive factor in identifying and responding to pressing urban needs, especially within a rapidly changing, desertifying and urbanized context like the U.S.-Mexico border region. For planning agencies and researchers serving binational borderland populations, however, access to complete, equal, and accessible data is hampered by an array of challenges. Researchers mining the multiple datascapes of the border region face unique informational challenges (gathering relevant, complete, and current data at adequate resolution and comparable scales), geophysical challenges (locating data in continuous and comparable cross-border geographies), and administrative challenges (making data available and affordable).

/Informational Challenges

Cross-border environments have long posed challenges for geospatial researchers constructing Geographic Information Systems (GIS) datasets in the borderland, significantly due to the heterogeneity of data sources available across jurisdictional divides. [1] Data fractures at the border depending on several factors [2] - we will focus here on formats, scales, and resolution.
Format

Data available in a given jurisdiction is conditioned by that jurisdiction's protocols and formatted to achieve its specific objectives. The two nations, ten states, and dozens of municipalities and administrative subdivisions that define the borderland constantly accumulate data relevant to borderspace studies, but have a range of approaches to how information is gathered, measured, and organized. Competing data models, languages, systems of measurement, and reporting frequencies are just a few of the formats which problematize the assembly of comparative, collective, and complete cross-border datasets.

\section{Scale}

There are significant differences in the scale of informational jurisdictions on both sides of the border, which can distort the grain of analysis and visualization they afford. The closest administrative analog for U.S. county boundaries in the Mexico system, for example, is the "municipio" or municipality. But municipios along the U.S.-Mexico border tend to be significantly smaller than their U.S. county counterparts, creating challenges for comparative studies in densities and locations of demographic and urban conditions.

\section{Resolution}

The grain, or resolution of data will vary depending on the density of data collection, both spatially and temporally. A loss of resolution can index disparities in the amount of expended resources, or participation in data regimes across jurisdictional divides. In one example, the resolution and availability of digital elevation model (DEM) information to produce a cross-border terrain model depends largely on "global" scale information supplied at a resolution of $30 \mathrm{~m}$ per pixel. Increased resolution is available from U.S. data sources but only within a certain distance from the border, creating a geopolitical gap in imaging both for each side of the border and for the contiguous region.

\section{/Geophysical Challenges}

Assuming equivalent information can be gathered across the divide, the next challenge is to geolocate that information within a set of coordinated geometries - the points, lines, polygons, and surface features of a GIS. Depending on the mapping source, surveying standards, and coordinate systems used to generate publicly available geospatial datasets-which 


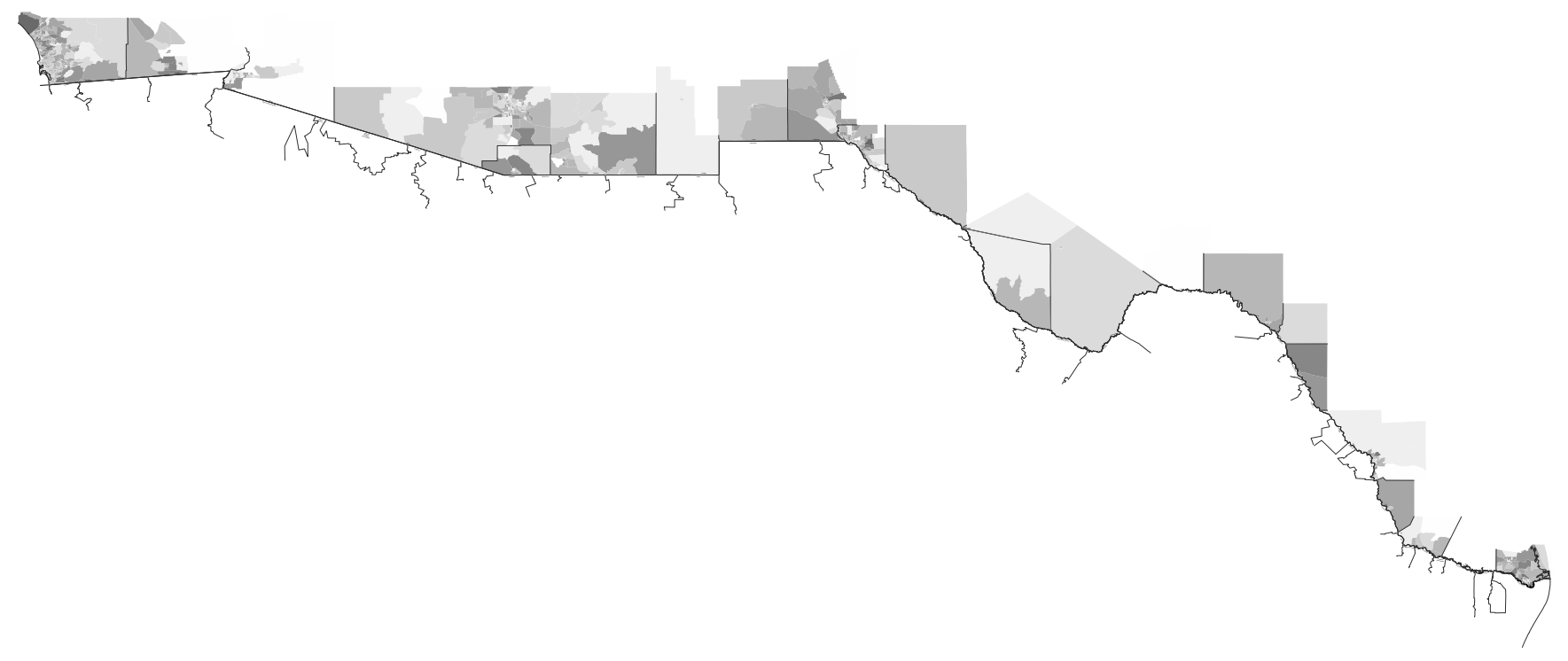

Figure 1. County and Municipio boundaries that fragment the U.S. - Mexico border region datascape and undermine its shared environmental and cultural conditions. Map by POST.

all can vary widely across the many informational regimes of the borderland-the mapped boundaries and limits of measured features will often contradict or overlap. The "loose fit" of assembled borderland geometries problematizes the accurate correlation of data to geolocated features. Edges blur in many ways, most commonly through misalignments and discontinuities.

\section{Alignment}

Misalignments occur when different data feature sources draw the extents of features differently. Varying degrees of precision, or contradicting assumptions about feature boundaries, can result in staggered boundaries of presumably continuous features, as when the boundary of an otherwise coherent ecological region "jumps" east or west as it crosses the border, or in multiple, competing interpretations of the same feature, as with the case of the national border. Despite its clearly-defined legal and physical boundaries, when rendered in geospatial analysis software the border can appear as fluid and dynamic as a meandering riverbed. Conflicting edges proliferate through borderspace depending on the source of the information and the acumen of its author.

\section{Continuity}

Discontinuities, "holes," and "blank spots," in maps of borderspace can result from a variety of factors, including unintentional gaps in the public data record, blindspots beyond the gaze of various remote sensing methodologies, and the censoring or withheld release of confidential geospatial data throughout the many regional spaces dedicated to national security and military installations. To smooth over the gaps of proprietary databases and public records, open source frameworks are promising alternatives, though in many border areas they can be as disjointed and incomplete as public or official sources. Open source topographic data from Open Street Map, for example, provides seemingly continuous cross-border elevation models, but on closer inspection the information is filled with substantial "holes." In the Paso del Norte region, significant topographic landmarks are absent, rendering Juárez a flat plain without its signature mountain ranges or piled foothills. Entire landscapes disappear within the datascape, victims of the national data divide.

\section{/Administrative Challenges}

The final-and perhaps most important-hurdle to establishing impactful and accurate transnational datasets is the complex and equally fragmented administration of the data. Assembling data from disparate jurisdictions and initiatives-some unilateral, others bilateral-depends on various forms of governance, institutions, and other data keepers to be present in the border region, concerned with its development, and promote data practices that are open, transparent, and accessible. In the geographic and political context of borderspace these criteria are not always met. The distance of border regions from administrative centers, the vulnerability of borderland data to changing political priorities, and systemic issues with access and affordability disrupt the easy flow of data to a borderland public.

\section{Remote Data}

Borderland territories are far from centers of governance. Border cities are remote from state and national capitals. In remote counties, vast geographies are characterized by low population density with limited resources, oversight and regulation, affecting the collection, archiving, processing, and access of information. 


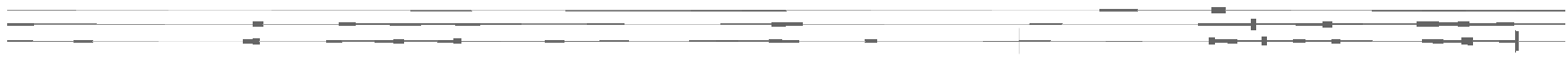

Figure 2. Index of Fragmentation, algorithmic drawing summarizing cross-border jurisdictional discrepancies along the US-Mexico border. Visualization by POST.

\section{Politicized Data}

Changing political climates and research agendas affect the availability of comprehensive cross-border environmental data. Information is gathered and held by mercurial entities whose priorities shift to political whims. For example, since the most recent US presidential election, access to crossborder data from the longstanding Border Environmental Health Initiative (BEHI) was made less accessible. The BEHI is a binational environmental geospatial database and interactive web interface managed by the U.S. Geological Survey (USGS), concerned with environmental degradation due to population density and land use changes. The database provides harmonized and continuous geospatial information addressing a range of geographies and phenomena of concern to the borderland, helping to visualize and analyze ecological impacts connected across otherwise unrelated administrative boundaries. Without continued access to the initiative's searchable webmap and linked geospatial files, researchers face a roadblock in mapping and understanding shared concerns.

Cycles of political structures also differ between neighboring municipalities along and across the border, creating other difficulties in the collection administration of data. In the Paso del Norte region, three-year cycles in Ciudad Juárez, and four-year cycles in El Paso, create mismatches in protocols and census priorities with each new administration.

\section{Inaccessible Data}

With limited platforms for shared, compiled cross-border data, researchers must become fluent in many complex, opaque, and constantly updating systems to gain the necessary information. Obtaining up-to-date census and national-level demographic data, for instance, demands a fluency in two national databases undergoing substantive shifts in their platforms for data provision. The US system is undergoing sweeping changes in preparation for the 2020 Census, decommissioning the long-standing web source, American Fact Finder. [3] Mexico's national administration for GIS data, Instituto Nacional de Estadística y Geografía (INEGI), has redeveloped its mapping and downloading service in the past few years, leaving the site in a "beta" version seemingly perpetually. Within the system, it is notoriously difficult to locate relevant data and download in continuous batches. To overcome these substantive accessibility issues, researchers have taken on the administrative task themselves, providing the research community with tools to circumvent the difficulties of opaque national platform. [4]

\section{INHABITING THE DATA BORDER}

To translate these informational ruptures into an immersive experience for Less Talk/More Action conference attendees, we developed an informationally driven film and audio environment. The installation, named Inhabiting the Data Border, delivered a deliberately cacophonous background for discussions around the informational and jurisdictional barriers that conference attendees face within their own academic contexts. The mapping, film, and audio components combine to exhaustively catalog and sequentially present the dozens of county and municipio boundaries that divide the datascape of the borderland.

\section{/Mapping: Index of Fragmentation}

From the perspective of any single county or municipio, we were interested in understanding how many different jurisdictions lie across the border, asking: how many different authorities would binational endeavors need to address? We developed a graphic index of fragmentation (Figure 2) for each jurisdiction, identifying those with the greatest challenges in assembling disparate populations and data sources to common goals.

\section{/Film: Scanning Data Disruptions}

The film scans this index of fragmentation like a radio dial tuning into areas of informational disparity, as the border geographies appear in tandem in a slowly scrolling crawl (Figure 3). The continuous scanning of the U.S.-Mexico border from its westernmost point near San Diego- Tijuana towards its eastern terminus in the Gulf of Mexico highlights the physical and political disjunctures that misalign every mile of the territory on both sides of the border.

\section{/Audio: Overlapping Authorities}

The audio track presents a series of overlapping, bureaucratic definitions of the territory and the features that divide it. We automated the generation of legal descriptions for each of the jurisdictional lines from the bearings and lengths stored in readily available geospatial data, and recorded audio using a text-to-speech reader. 


\section{/EDUCATING ACROSS DATA BORDERS}

As architectural educators operating in the borderland, we confront the data border daily with our students. In this unique educational context, in a vibrant binational culture bursting with new forms of binational knowledge, accessing, processing, and sharing that knowledge faces economic barriers. Here we are working to increase access to the equipment, software, tools, and instruction for our students to understand and overcome the data border.
We are also working to provide platforms for increased collaboration in cross-border research across the many institutional and individual assets of the region. Demographic and socioeconomic mappings of the border region reveal an uneven attainment of college-level education in cross-border counties throughout the region (Figure 4). Educational and economic disparities between "sister cities" and cross-border counties set up entry barriers to informational equity. The U.S. side of the border has a higher concentration of college-educated

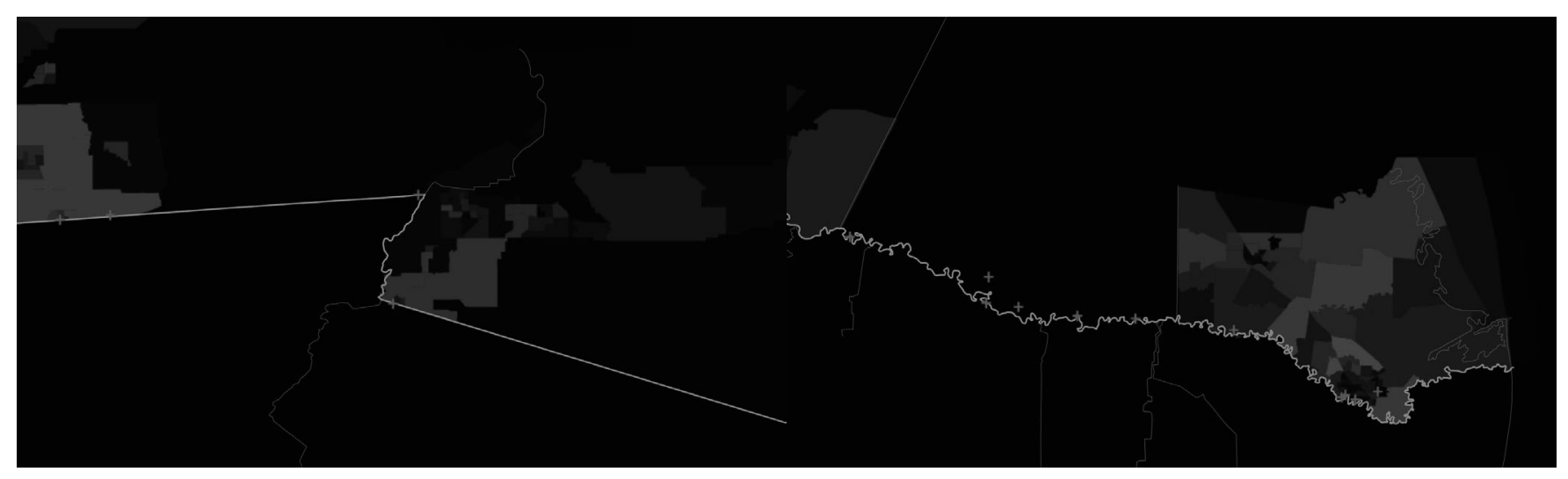

Figure 3: Details, Inhabiting the Data Border Film. Image Credit: POST

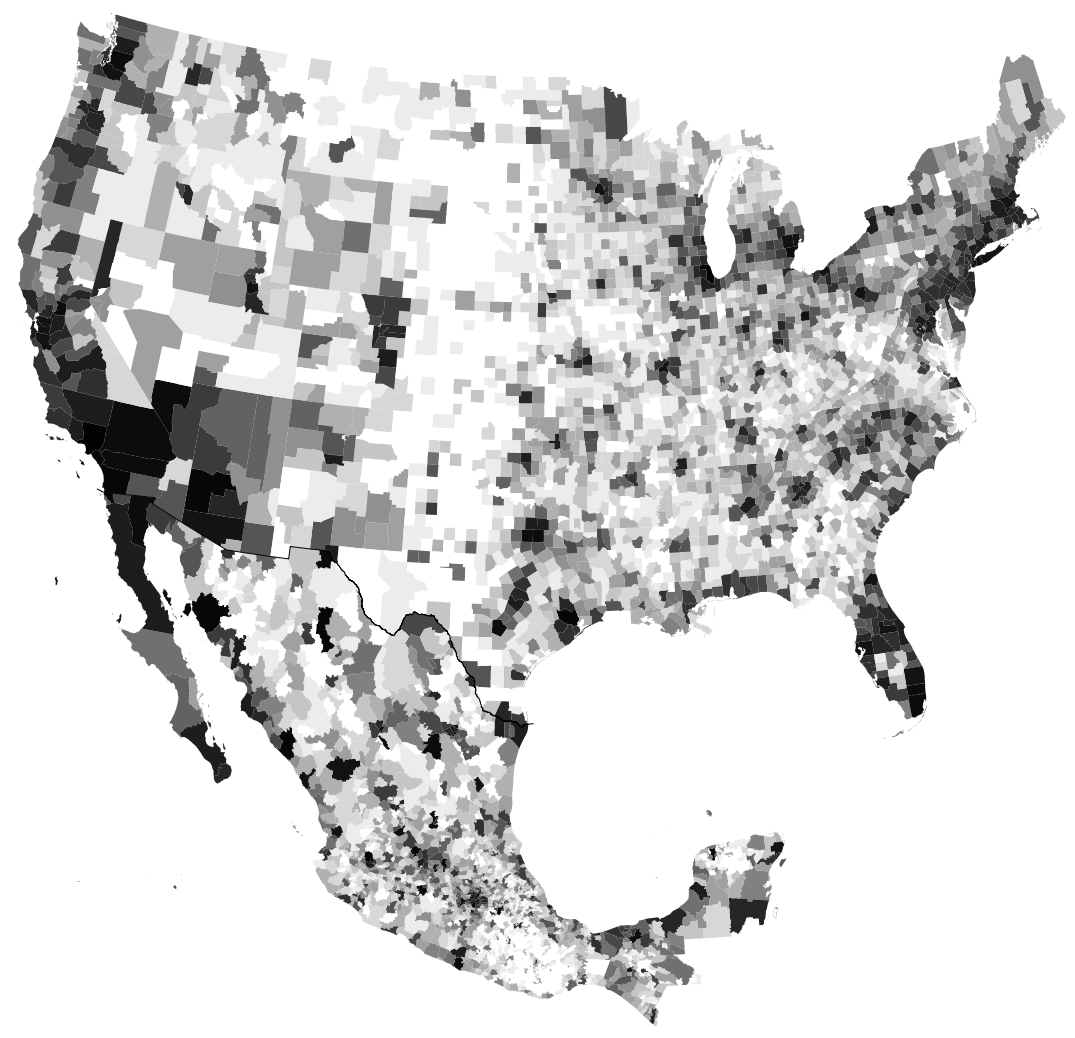


individuals. While there is a fair amount of universities, architecture degree programs, and other robust research bodies along the border region, we see opportunities for more crossborder schools and pedagogies. Research about the shared context is developed and concentrated largely within institutional boundaries, but researchers are increasingly seeking audiences beyond the walls of the academy and across the international divide.

/POST - Project for Operative Spatial Technologies

The increasingly contested U.S.-Mexico border region calls for new design, research, and visioning methods enacting impact at territorial scales. We are leading students and engaging educational partners in these issues as we direct POST (Project for Operative Spatial Technologies), an experimental, investigative, territorial think-tank situated on the border, which leverages emerging spatial technologies to anticipate transformations in urbanization, land use, and resource depletion in binational desert territories. [] The research center is housed within the College of Architecture at Texas Tech University's El Paso campus, steps away from the U.S.-Mexico border. POST develops datasets, visualizations, and analytical tools using emerging spatial and geospatial technologies.

We develop platforms for collaborative research across institutions and disciplines, and engage undergraduate researchers through our work. The strategic geopolitical grounding is the ideal location for applied research in emerging spatial technologies.

The work of the center invigorates our teaching and contributes to the school's unique educational platform. Carefully considered regional issues serve as starting points for design endeavors in which students are empowered to identify, visualize, and respond to pressing environmental issues impacting binational desert cities, and prepare them for productive roles in the region and beyond. Students actively measure, draw, and imagine the territory with a critical eye towards jurisdictional fragmentation which effectively marginalizes their context.
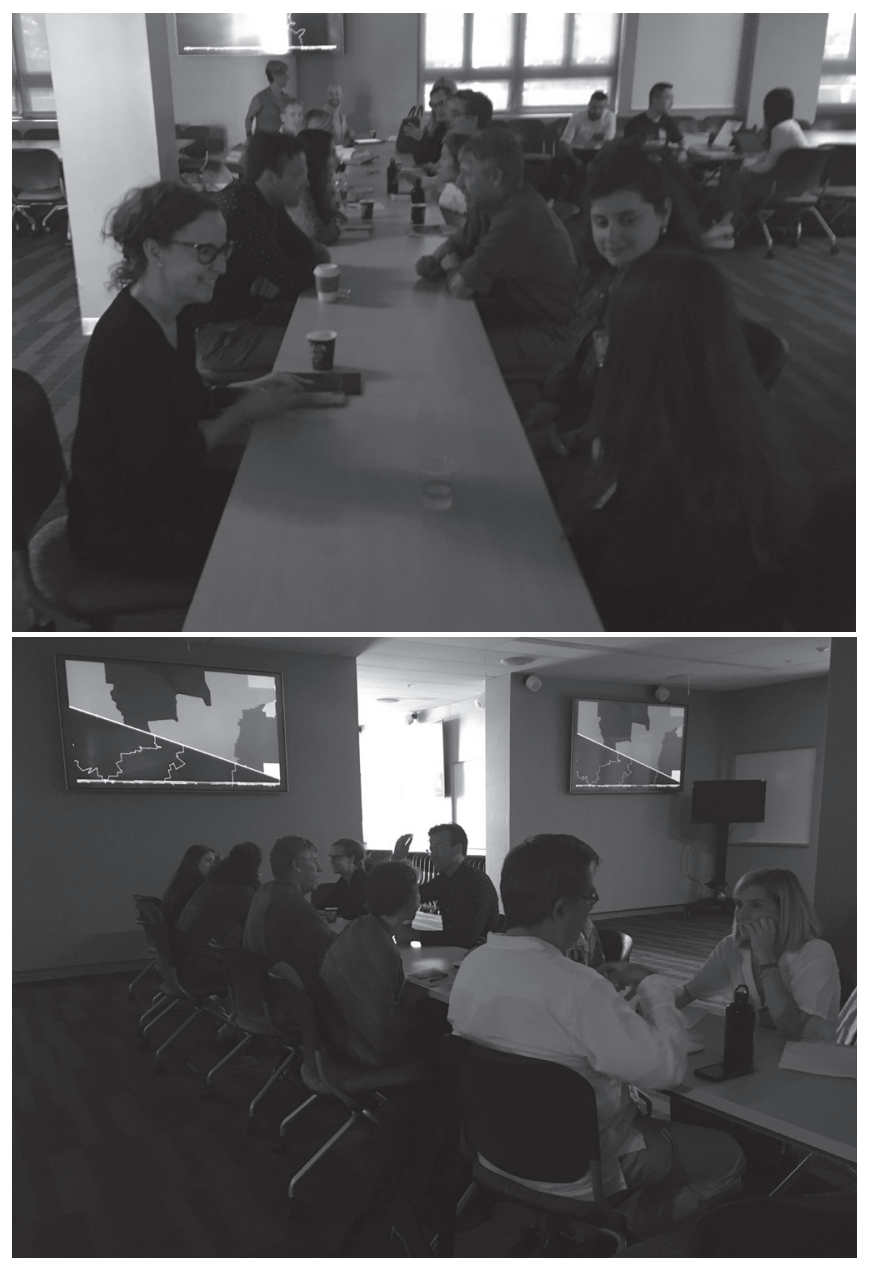

Figure 5: Inhabiting the Data Border at ACSA Stanford, Less Talk/More Action

\section{ENDNOTES}

1. See, e.g., Paso del Norte Water Task Force. "Creating A Single Map Regional Geographic Information System to Support Water Planning In The Paso Del Norte Region." (2002).

2. See, e.g., http://www.fig.net/resources/proceedings/fig_proceedings/ fig2006/papers/ts82/ts82_04_donaubauer_etal_0603.pdf

3. See https://data.census.gov/cedsci/deeplinks?url=https\%3A\%2F\%2Ffactfin der2.census.gov\%2F\&tid=GOVSTIMESERIES.CG00ORG01

4. Diego Valle Jones has developed one such datasource, which has compiled INEGI data of the most recent geoestadistico framework for batch download. See Diego Valle Jones, https://www.diegovalle.net

5. See Project for Operative Spatial Technologies, https://www.post.arch.ttu.edu 